\section{Lillian Clark}

is senior lecturer at University of Portsmouth Business School. She is course leader for the MA Digital Marketing programme, lectures in digital marketing and supervises PhD/DBA students. Her research interests include online consumer behaviour, culture and digital marketing, online political marketing, and the use of social media in $B 2 B$ activities.

Keywords: digital marketing, market research, data protection

New areas of research

\section{Privacy and data ownership}

Lillian Clark

Portsmouth Business School, University of Portsmouth, Richmond Building, Portland Street, Portsmouth PO1 3DE, UK.

Tel: +44 (0)239284 4820

E-mail: lillian.clark@port.ac.uk

\section{Opinion Piece}

\section{Ethical marketing research in the digital age - How can academics and practitioners work together?}

\author{
Lillian Clark
}

\begin{abstract}
This piece considers how the experience of marketing practitioners and academics can be pooled to overcome ethical objections to certain standard market research practices in the digital world. Journal of Direct, Data and Digital Marketing Practice (2014) 15, 258-259. doi:10.1057/dddmp.2014.17
\end{abstract}

'And what is good, Phaedrus, and what is not good - need we ask anyone to tell us these things?' (R. Pirsig, Zen and the Art of Motorcycle Maintenance)

Like everything else in its path, the advent of interactive digital technologies has transformed research, both for marketing academics and practitioners. Not only have tools such as online surveys, email interviews and online focus groups provided wider access to participants and quicker results, but entirely new areas of research have sprung up. Topics such as online consumer behaviour, engagement with social media and responses to smartphone advertising have become areas of profound interest to the marketing academic and practitioner alike.

However, digital technologies have also brought new challenges to doing research in an ethical manner. Questions of privacy and confidentiality, reliability of data collected and distribution of results have become problematic in a digital world where people can perceive they are hiding their identity but actually leave traces of their activities and intentions, and where the rallying cry of the internet in the 1990s — 'information wants to be free' — has since become the perception that everything available online has no fee or ownership.

Adding to these challenges are pressures from legal and regulatory bodies. For example, UK practitioners have to deal with the implementation of the so-called 'Cookie Law' of 2011, which requires sites to explicitly obtain consent from users before storing or retrieving information on their device, but it is unclear whether users actually understand what they are giving consent to. Other legislation designed to protect children from inappropriate advertising, or requiring permission to obtain or use personal data, struggle to keep up with the latest technologies, but must still be taken into consideration. 


\section{Research subject to ethical review}

\section{Do incentives violate anonymity?}

\section{Ethical research is good research}

\author{
Increasing \\ co-operation increases \\ value
}

For academic researchers, another pressure to consider is the institutional Ethical Review process. Research councils, universities and journals are now insisting on ethical review of research as a condition of funding and/or publication. This review process is designed to ensure ethical compliance, rather than judge the quality or suitability of the proposed research. But digital research is coming under increased scrutiny, raising issues that research institutions are unsure how to cope with, or which are in conflict with practitioner norms.

The key to addressing these issues lies in practitioners and academics sharing education and expertise to understand and address the ethical challenges inherent in online market research. Take, for example, the question of remunerating participants in online research. Practitioners are accustomed to providing incentives to encourage online survey completion, usually via prize draws. However, some institutional ethics review panels are opposed to this practice, claiming that this would violate principles of anonymity as the participant would have to provide contact details in order to be eligible for a prize, and that, in fact, privacy concerns may actually preclude participation if an email address needs to be provided. (In practice, ethical concerns about participation incentives can be addressed by storing contact data separately from survey responses, and ensuring that separation is made clear at the start of the survey.)

This is something that both academics and practitioners can easily implement, and should be standard practice when offering incentives for online surveys. The concern about incentives being counter-productive needs to be challenged and challenged strongly. While there is not a preponderance of academic studies on this topic, what is out there does suggest that incentivising participants is a useful practice in online surveys and one in which academics and practitioners should be able to work together to demonstrate its efficacy.

This sharing of education and expertise is not a one-way street, as practitioners can use their experience to assist the academic in solving ethical issues. For example, academics often have an extensive background in research ethics and should therefore be in an ideal position to educate the practitioner on why 'ethical research is good research', and how to identify potential ethical problems and develop appropriate solutions. This type of training would be an invaluable contribution to any certification in digital marketing, including the IDM's own diploma programmes.

In a previous issue of this journal, Professor Merlin Stone argued that academics need to make their research more relevant to practitioners. Co-operation in understanding and responding to the ethical challenges inherent in digital research can not only increase the value of academic research to the business community, but also bring the value of practitioner research to the academic world.

\section{Reference}

Merlin, S. (2013) 'Co-operation between academics and practitioners - Hope for the future?', Journal of Direct, Data and Digital Marketing Practice, Vol. 15, No. 2, pp. 105-107, doi:10.1057/dddmp.2013.54. 